\title{
A meta-analysis of re-treatment for intravenous immunoglobulin-resistant Kawasaki disease
}

First published online: 23 April 2015

Dear Sir,

I read with interest the paper entitled "A metaanalysis of re-treatment for intravenous immunoglobulin-resistant Kawasaki disease". I have the following comments to offer. The risk of publication bias has not been discussed, which has a great bearing on the interpretation of the results. It may cause skewed results, especially towards positive outcomes. A funnel plot is used for the purpose. Second, the results of the meta-analysis are limited to the four studies on which it has been conducted. Although authors have not reported which model - fixed or random effects model - they have used for the analysis, it appears from the software output that it is the "fixed effects model". Most authors are now using the "random effects model" because the results can be applied to a larger set of studies, from which the four studies under investigation have been taken. The fixed effect model is appropriate in the absence of moderators, any patient or study design characteristic, which can affect the results. I agree that it is difficult to see/foresee many such moderators. The difference in the design of the studies selected - two randomised, two non-randomised, and type of allocation - may act as a moderator. ${ }^{2}$ Therefore, I opine that a random effects model would have been appropriate, or if a fixed effects model is used the drawbacks of it should have been discussed.

Sudha Chandelia

Department of Pediatrics, Division of Pediatric Critical Care, PGIMER and Associated Dr. Ram Manohar

Lobia Hospital, New Delbi, India

\section{References}

1. Yang X, Liu G, Huang Y, et al. A meta-analysis of re-treatment for intravenous immunoglobulin-resistant Kawasaki disease. Cardiol Young 2015.

2. Hedges LV, Vevea JL. Fixed and random effects models in meta-analysis. Psychol Methods 1998; 3: 486-504. 